\title{
Nano-scale measurement of biomolecules by optical microscopy and semiconductor nanoparticles
}

\author{
Laboratory for Comprehensive Bioimaging, RIKEN Quantitative Biology Center, Suita, Osaka, Japan \\ ${ }^{2}$ Laboratory for Nano-Bio Probes, RIKEN Quantitative Biology Center, Suita, Osaka, Japan \\ ${ }^{3}$ Graduate School of Frontier Biosciences, Osaka University, Suita, Osaka, Japan \\ ${ }^{4} \mathrm{WPI}$, Immunology Frontier Research Center, Osaka University, Suita, Osaka, Japan \\ ${ }^{5}$ Department of Physics, School of Science, The University of Tokyo, Bunkyo, Tokyo, Japan
}

Taro Ichimura1, Takashi Jin ${ }^{2,3,4}$, Hideaki Fujita ${ }^{1,4}$, Hideo Higuchi' ${ }^{5}$ and Tomonobu M. Watanabe ${ }^{1,3,4 *}$

\section{Edited by:}

Rinshi S. Kasai, Institute for Frontier Medical Sciences, Japan

\section{Reviewed by:}

Takahiro Fujiwara, Kyoto University, Japan

Yajima Junichiro, Tokyo, Japan

\section{*Correspondence:}

Tomonobu M. Watanabe, Laboratory for Comprehensive Bioimaging, RIKEN Quantitative Biology Center, 6-2-3, Furuie-dai, Suita, Osaka 565-0874, Japan

e-mail: tomowatanabe@riken.jp
Over the past decade, great developments in optical microscopy have made this technology increasingly compatible with biological studies. Fluorescence microscopy has especially contributed to investigating the dynamic behaviors of live specimens and can now resolve objects with nanometer precision and resolution due to super-resolution imaging. Additionally, single particle tracking provides information on the dynamics of individual proteins at the nanometer scale both in vitro and in cells. Complementing advances in microscopy technologies has been the development of fluorescent probes. The quantum dot, a semi-conductor fluorescent nanoparticle, is particularly suitable for single particle tracking and super-resolution imaging. This article overviews the principles of single particle tracking and super resolution along with describing their application to the nanometer measurement/observation of biological systems when combined with quantum dot technologies.

Keywords: single particle tracking, super-resolution, fluorescent microscopy, quantum dot, nanoparticle

\section{INTRODUCTION}

Fluorescence microscopy has become standard for studying the dynamic behavior of biological phenomena such as the expression, movement, and localization of proteins and other molecules (Ellinger, 1940; Lichtman and Conchello, 2005; Drummen, 2012; Miyawaki, 2013; Peter et al., 2014). Optical diffraction, however, limits the spatial resolution to several 100 nanometers, denying information on many details about these phenomena (Abbe, 1873). Two technologies have since overcome this limitation and permit the observation of even smaller nano-scale dynamics: single particle tracking (Ritchie and Kusumi, 2003; Saxton, 2009; Chenouard et al., 2014) and super-resolution microscopy (Schermelleh et al., 2010; Galbraith and Galbraith, 2011; Leung and Chou, 2011). Single particle tracking pursues the position of single fluorescent probes conjugated to separate target proteins over a two-dimensional (2D) plane. Super-resolution microscopy, on the other hand, provides highly resolved optical images beyond the aforementioned spatial resolution.

To conduct the above imaging techniques, it is often required to label the target protein with a fluorescent probe. Fluorescent proteins are most popular for this purpose because of their simple and easy labeling procedure in live cells (Shimomura and Johnson, 1692; Tsien, 1998; Nifosí et al., 2007). Organic dyes are also common because of their wide application (Wombacher and Cornish, 2011; Wysocki and Lavis, 2011; Terai and Nagano, 2013). Another group of probes gaining attention is inorganic nanoparticles made of semiconductors, metals, silicon, etc. (Ruedas-Rama et al., 2012; Chinnathambi et al., 2014; Cupaioli et al., 2014).
Although usually larger than fluorescent proteins and organic dyes, inorganic nanoparticles have generally stronger and more stable fluorescence profiles, which makes them applicable not only to basic research, but also to clinical studies (Byers and Hitchman, 2010; Choi and Frangioni, 2010; Saadeh et al., 2014; Wang and Wang, 2014). Furthermore, these same properties make them well suited for single particle tracking methods (Chang et al., 2008; Saxton, 2008; Barroso, 2011; Bruchez, 2011; Clausen and Lagerholm, 2011; Ruthardt et al., 2011; Pierobon and Cappello, 2012; Kairdolf et al., 2013; Petryayeva et al., 2013).

This review article focuses on advanced microscopy using quantum dots (Qdots), perhaps the most studied of inorganic nanoparticles for biological application (Pilla et al., 2012). Single particle tracking using Qdots has reached three dimensions (X, Y, Z) (Genovesio et al., 2006; Holtzer et al., 2007; Watanabe and Higuchi, 2007; Watanabe et al., 2007; Ram et al., 2008, 2012; Wells et al., 2008, 2010; Yajima et al., 2008), and more recently has even reached four dimensions (X, Y, Z, $\theta$ or X, Y, $\theta, \varphi$ ) (Ohmachi et al., 2012; Watanabe et al., 2013). For all their benefits, Qdots do have drawbacks, however, including high blinking (Nirmal et al., 1996; van Sark et al., 2001; Schlegel et al., 2002; Hohng and Ha, 2004; Ko et al., 2011) and a spectral blue-shift during observation (Nirmal et al., 1996; van Sark et al., 2002; Hoyer et al., 2011), which complicate the continuous tracking of the single particle and emerge due to photo-oxidation while under high-power illumination. These limitations have stimulated research into new super-resolution microscopy methods (Lidke et al., 2005; Dertinger et al., 2009; Watanabe et al., 2010; Chien et al., 2011; Hoyer et al., 2011; Deng et al., 2014). 


\section{Qdots AS FLUORESCENT LABELING PROBES}

A Qdot is a semiconductor nanocrystal with electronic characteristics that depend on its size and shape (Rossetti et al., 1980; Ekimov and Onushchenko, 1981). Because of its unique characteristics and ease of synthesis, Qdots have been applied not only to biomedical research, but also to engineering- and industrialrelated fields such as transistors, solar cells, LEDs, and diode lasers (Pilla et al., 2012). Qdots used in biological studies have a core-shell structure (Figure 1A); the most famous being the cadmium selenide (CdSe) core and zinc sulfide (ZnS) shell (Dabbousi et al., 1997; Bruchez et al., 1998; Chan and Nie, 1998; Pilla et al., 2012). This structure results in Qdots having narrow emission spectra but wide absorption spectra (Figure 1B). There are two important criteria when applying Qdots to biological studies: solubility and conjugating capability (Li et al., 2010). Highly fluorescent Qdots are usually synthesized in organic solvents in coordination with compounds such as tri-n-octylphosphine oxide (TOPO) or alkylamine. These compounds coat the Qdot, making it too hydrophobic to be dissolved in water. Therefore, further surface coating or exchange with hydrophilic compounds is needed for use in biological assay. Furthermore, upon becoming water soluble, the surface of the Qdot must have reactive groups such as amino and carboxyl chains in order for the Qdot to conjugate with the target biological sample. The surface coating contributes not only to the water-solubilization but also to the stabilization of the fluorescence of Qdot in water because the

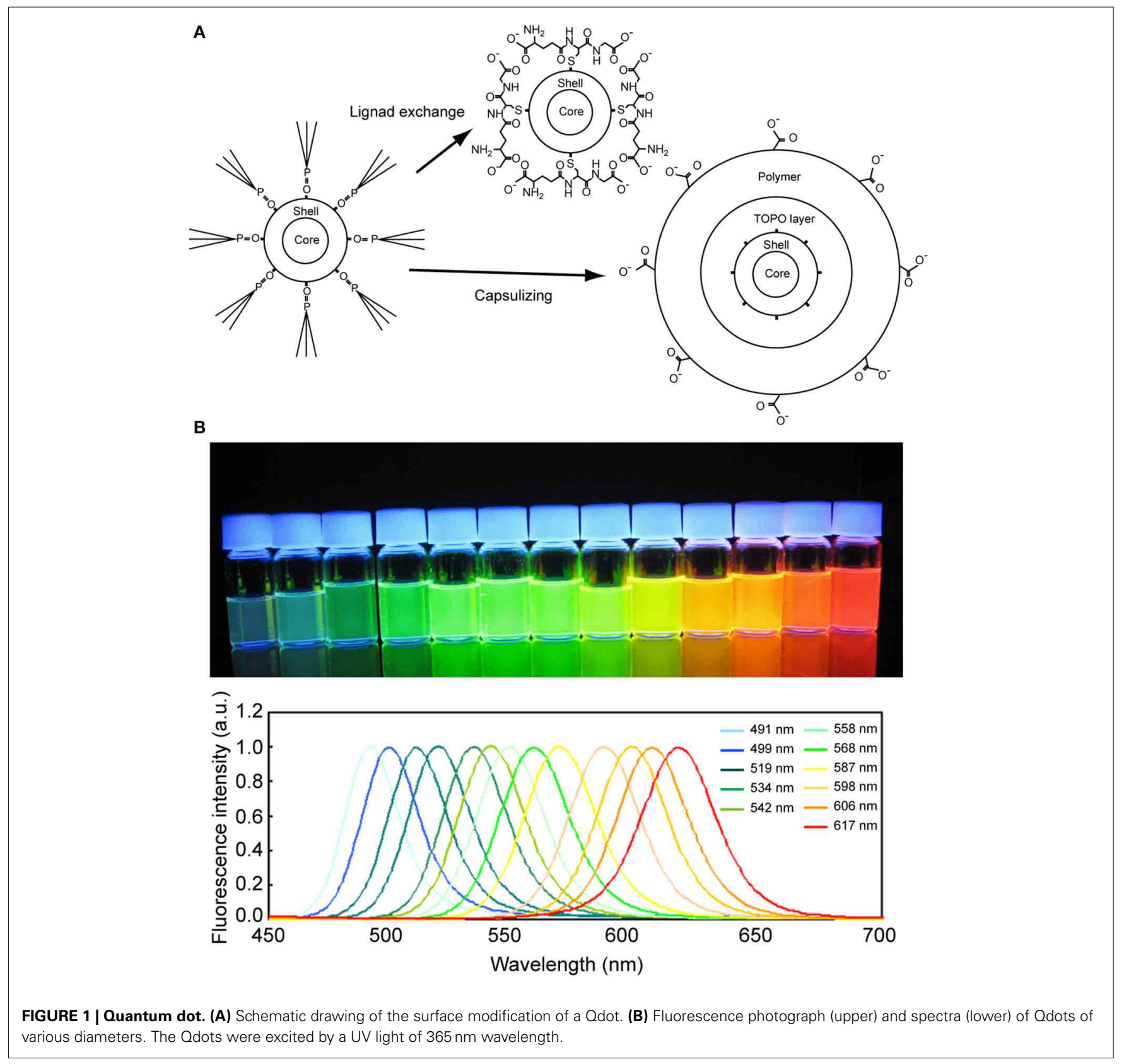


photophysical properties are well affected by the surface coating (Kuno et al., 1997; Kloepfer et al., 2005). Some surface coating methods suppress the blinking that is a drawback of Qdot (Hohng and Ha, 2004; Fomenko and Nesbitt, 2008; Mandal and Tamai, 2011; Zhang et al., 2013).

There are mainly two ways to prepare water-soluble Qdots (Figure 1A) (Erathodiyil and Ying, 2011; Zhang and Clapp, 2011). The first is to encapsulate a hydrophobic Qdot with an amphiphilic polymer or phospholipid (Dubertret et al., 2002; Gao et al., 2005; Li et al., 2010; Tomczak et al., 2013). The second is a ligand-exchange method in which the capping hydrophobic ligands are exchanged with hydrophilic ones (Gerion et al., 2001; Guo et al., 2003; Pinaud et al., 2004; Kim et al., 2005; Nann, 2005; Jiang et al., 2006; Dubois et al., 2007). While the water-solubilized Qdot obtained by the first method is more stable and suitable for commercialization, its size increases to about $20 \sim 40 \mathrm{~nm}$, which risks steric hindrance against the function of the target protein (Li et al., 2010). The ligand-exchange method is inferior in stability, but is a simpler synthesis process and produces a smaller Qdot. The thin coating layer is another advantage of this method, as it reduces the risk of steric effects that could compromise the function of the protein upon conjugation with the Qdot.

Many coating agents exist for the ligand-exchange method. These include mercaptocarboxylic acid (Jiang et al., 2006), carbon disulfide (Dubois et al., 2007), thiosilanol (Gerion et al., 2001), dendrimer (Guo et al., 2003), peptide (Pinaud et al., 2004), phosphine oxide (Kim et al., 2005), and polyethylenimine (Nann, 2005). Coating agents can also sometimes functionalize Qdots for specific purposes. Examples include $\beta$-cyclodextrin for ionsensing (Palaniappan et al., 2004), cyclodextrin for redox-active substrates (Palaniappan et al., 2006), and cyclodextrin thiol for pH-sensing (Cao et al., 2006). We usually use glutathione as the coating compound because of its easier preparation, which requires only the mixing of hydrophobic Qdots with an aqueous glutathione solution (Jin et al., 2008; Tiwari et al., 2009). Glutathione-coated Qdots have two reactive groups (amino and carboxyl) that enable easy conjugation with the target protein and show no cytotoxity (Tiwari et al., 2009). They can also be kept mono-dispersed in solution for 3 months after solubilization.

\section{FLUORESCENCE MICROSCOPY FOR NANO-SCALE MEASUREMENTS/OBSERVATIONS}

The microscopy introduced in this review requires a regular wide-field fluorescence microscope and no complicated optical principles nor devices (Figure 2A). However, because nano-scale measurements require a high signal-to-noise ratio, a highly photon-sensitive camera, such as an electron multiplying charge coupled device (EMCCD) camera, is recommended. More recently, complementary metal-oxide-semiconductor (sCMOS) cameras have become available as alternatives (Huang et al., 2011; Long et al., 2012; Ma et al., 2013). The vibration and/or stage drift of the microscope should also be considered, as these can cause artifacts in the measurement by obscuring the behavior and structure of the target. Consequently, the microscope should be set on a vibration-isolation table and built with as minimal height and maximal rigidity as possible to decrease any vibration. Because thermal expansion of the metals composing the

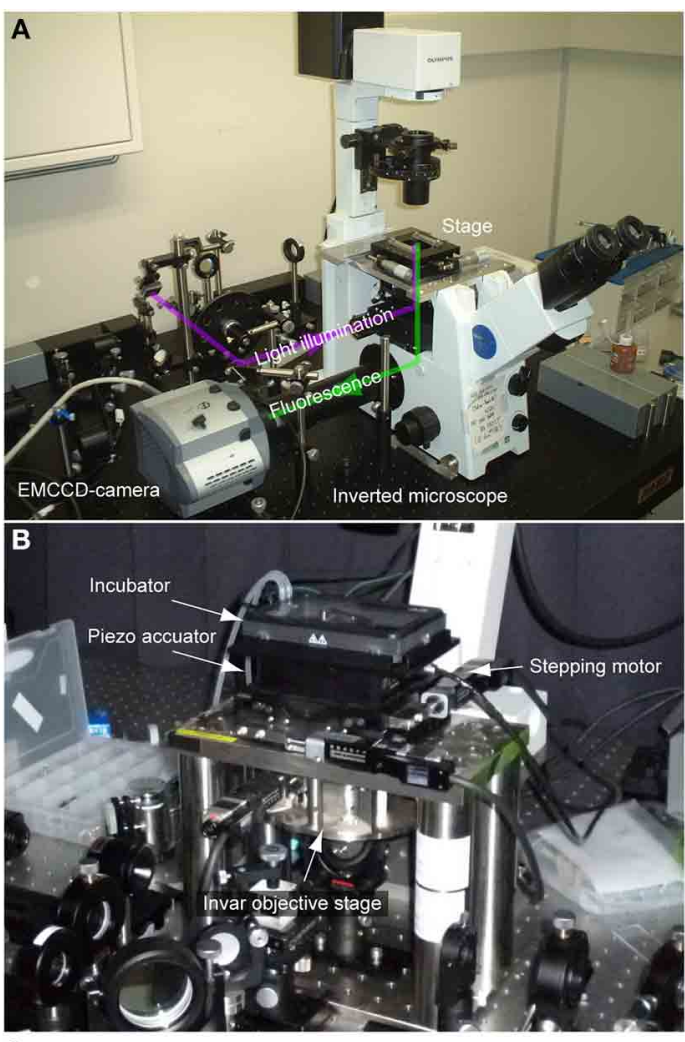

C
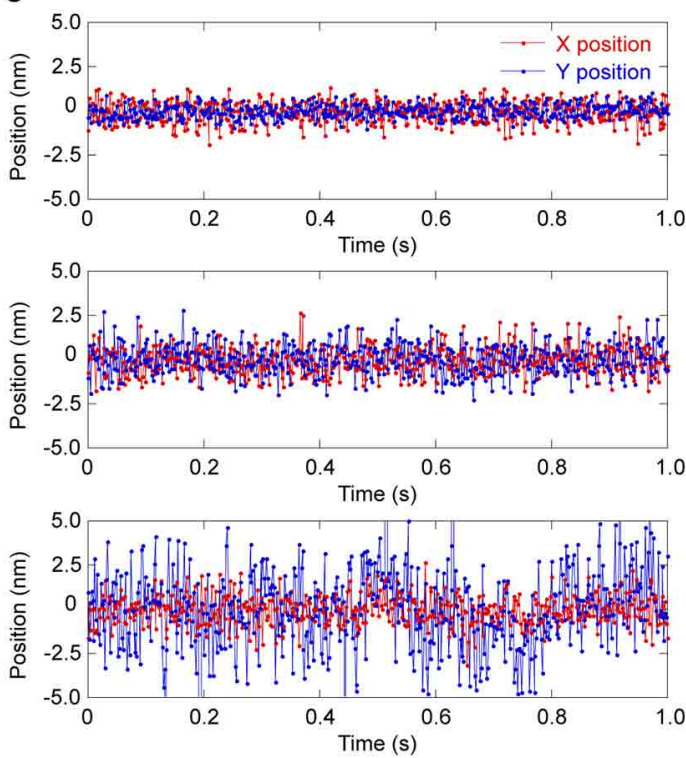

FIGURE 2 | Microscopic system for nanometery. (A) Photograph of a typical microscopy setup. The system is mainly composed of an inverted fluorescent microscope (Olympus IX71), an objective lens (Olympus 60x PlanApo, 1.45 NA, oil immersion) and EMCCD camera (Andor iXon887 or 867). (B) The stage and objective revolver are made of duralumin and custom built. (C) Vibrations caused by different components of the microscope: all components rigidly fixed (upper), all components except the camera rigidly fixed (middle), and substitution of the mono-objective revolver with a 6-position revolver (lower). Red, X-position. Blue, Y-position. The stage position was determined by measuring the position of a glass bead absorbed on the sample surface. 
microscope causes drifts in the stage and focus position, microscopes made of metals with lower thermal expansion such as invar are generally preferred (Figure 2B). The drifts can be further suppressed by setting the microscopic system in a room with constant temperature and humidity.

Here we show one strategy for reducing vibrations. The transition images of a silica bead with $1 \mu \mathrm{m}$ diameter absorbed on a coverslip surface were acquired with excess illumination so that the camera gain could be set to zero. The frame rate was $2.0 \mathrm{~ms}$, the images were acquired for $1.0 \mathrm{~s}$, and the precise position $(\mathrm{X}, \mathrm{Y})$ of the bead was calculated by image analysis. In our usual setup, the position of the bead was kept stable within $0.7 \mathrm{~nm}$ in the $\mathrm{X}$-axis and $0.4 \mathrm{~nm}$ in the Yaxis (Figure 2C, upper). When a screw to fix the CCD camera was loosened, the vibration increased to $0.8 \mathrm{~nm}$ in both axes (Figure 2C, middle). Normally, we use a mono-objective revolver, but when instead a commercially-available 6-position revolver was used, we found the vibration enhanced in the Yaxis to $2 \mathrm{~nm}$ (Figure 2C, bottom). Thus, rigid construction of the microscope is paramount for nano-scale measurements and observations.

\section{SINGLE PARTICLE TRACKING WITH NANOMETER PRECISION USING Odots}

Single particle tracking is well applied for studies of motor proteins and membrane proteins, because resolving nano-scale movements is necessary for understanding the protein function (Ritchie and Kusumi, 2003; Park et al., 2007; Toprak and Selvin, 2007; Saxton, 2009). Although the resolution of conventional fluorescence microscopes is constrained by the diffraction limit, the $2 \mathrm{D}$ position of a single particle can be determined by calculating the weight center of the image of the fluorescent spot (Figure 3A). The fluorescence emitted from a fluorescent probe forms a point spread function (PSF) that can be fitted with a Gaussian distribution as

$$
f(x, y)=I_{0} \cdot \exp \left\{-\frac{\left(x-x_{0}\right)^{2}+\left(y-y_{0}\right)^{2}}{2 \cdot \sigma^{2}}\right\}+C
$$

where $I_{0}$ and $\left(x_{0}, y_{0}\right)$ are the fluorescence intensity and the position of the fluorescing center, respectively, $\sigma$ is the radial standard deviation of the Gaussian function, and $C$ is the background fluorescence. This analysis can be used to measure the center position of the image (Kubitscheck et al., 2000; Cheezum et al., 2001; Thompson et al., 2002; Small and Stahlheber, 2014). Though there are other common methods for determining the center, including cross-correlation, sum-absolute difference, and simple centroid, Gaussian fitting has the highest robustness at low signal-to-noise ratios, which is common in biological studies (Thompson et al., 2002). In our case, the actual fitting computation is done by the Levenberg-Marquardt method (Levenberg, 1944). A practical example of our fitting is described below (Figure 3B). Because the background baseline is not always uniform in live-cell observations, we added additional parameters into the $C$ term to fit the local background fluorescence with a tilted plane,

$$
\begin{aligned}
f(x, y)= & I_{0} \cdot \exp \left\{-\frac{\left(x-x_{0}\right)^{2}+\left(y-y_{0}\right)^{2}}{2 \cdot \sigma^{2}}\right\} \\
& +C_{0}+C_{1} x+C_{2} y .
\end{aligned}
$$

This equation assumes the small area inside the region of interest (ROI) can be approximated by the plane. The initial parameters of the fitting are calculated by the linear least-square method for $C_{0}$, $C_{1}$, and $C_{2}$ using only the outer boundary of the ROI. Because the logarithm of the subtraction between $f\left(x_{0}, y_{0}\right)$ and $C_{0}+C_{1} x+$ $C_{2} y$ is a simple quadratic function, the other initial parameters are obtained by the linear least-square method, too. Setting the initial values close to the true values by these simple pre-calculations allows us to effectively reduce the number of the loop iterations in the Levenberg-Marquardt method.

The calculation precision by Gaussian fitting strongly depends on the photon number that the detection device receives from the emission of the fluorescent probe and can be as small as a few nanometers (Figure 3C) (Deschout et al., 2014; Small and Stahlheber, 2014). The method described above is called fluorescence imaging with one-nanometer accuracy (FIONA) and has quickly become a standard in the field (Yildiz et al., 2003; Yildiz and Selvin, 2005; Park et al., 2007; Hoffman et al., 2011). However, the number of photons emitted by single organic dyes and fluorescent protein molecules before photobleaching, about 110,000 (Kubitscheck et al., 2000), is too low for the observation of protein movement over a long time. Since the cause of photobleaching is thought to be oxygen collisions with the dye molecule in its excited state, it can be mitigated by the addition of oxygen scavengers (Sambongi et al., 1999; Adachi et al., 2000). Thus, the photon number from a single dye molecule can be increased to 1.4 million photons before photobleaching (Yildiz and Selvin, 2005). Meanwhile, Qdots show slight photobleaching and strong fluorescence even in the absence of scavengers (Bruchez et al., 1998). Though non-fluorescent nano-particles such as gold nano-particles are becoming increasingly popular for precise and long-term tracking using absorption (Kusumi et al., 2005; Lasne et al., 2006) or scattering (Nishikawa et al., 2010), the Qdot is still preferred in biological studies because of its wider color spectrum.

We investigated the relationship between the tracking precision and the average number of photons emitted from a Qdot (Figure 3C). The tracking precision was defined as the standard deviation of 100 data obtained with a Qdot immobilized on a glass surface in our case. While the experimental accuracy was a little lower than the theoretical expectation because of high blinking, it was still $2 \mathrm{~nm}$ when the photon number from a Qdot was 15,000 per exposure. To demonstrate the potential of single particle tracking as a biological tool, we measured the movement of kinesin, a microtubule-mediated motor protein (Figure 3D). The motor domain of the kinesin was fused with biotin career protein (BCCP) and conjugated with a Qdot via biotin-avidin affinity. The Qdot-labeled kinesin were then bound to microtubules adsorbed onto a cover slip. Upon adding $1 \mathrm{mM}$ ATP, the Qdot was seen to move unidirectionally along the microtubule without detaching, which is consistent with kinesin using ATP to move (Figure 3D, left). The unidirectional movement of kinesin 


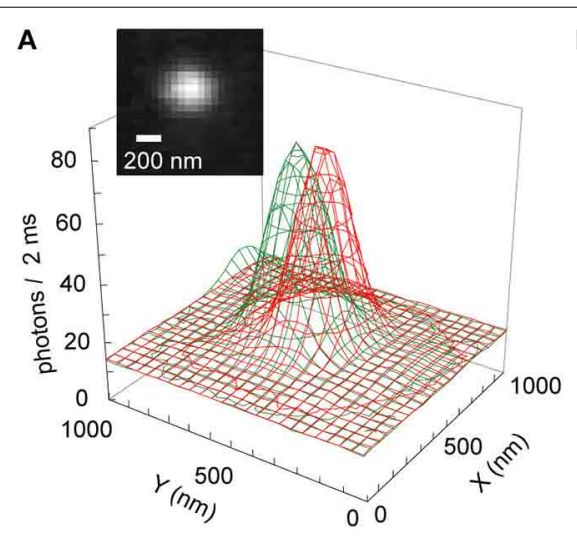

B Fluorescence image
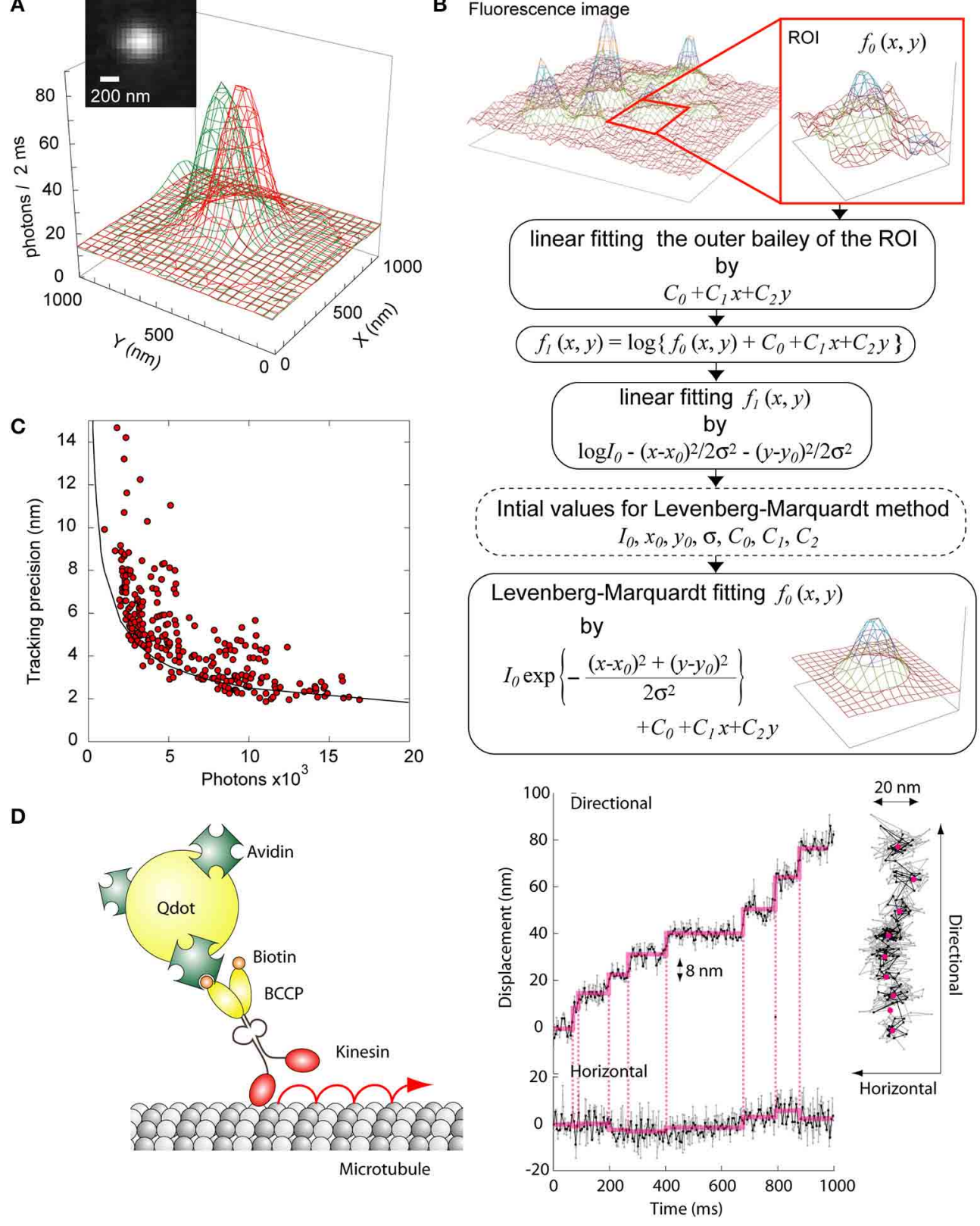

FIGURE 3 | Single particle tracking. (A) Fluorescence profiles of a Qdot bound to a moving motor protein. The green-colored profile was taken at time 0 and the red profile at $t=2 \mathrm{~s}$. The difference of the peaks of the two profiles was about $200 \mathrm{~nm}$. (Inset) Fluorescent image of the Qdot. (B) Calculation scheme to determine the center position of a Qdot. (C) Relationship between

the tracking precision by Gaussian fitting with the number of photons emitted from a single fluorophore. Circles, experimental data. Line, theoretical value (88). (D) Single particle tracking of kinesin. Left, schematic of the assay. Right, typical trace of a single kinesin. The frame rate of the image acquisition was $2 \mathrm{~ms}$. The photon number emitted from the Qdot was about 10,000.

was composed of successive $8 \mathrm{~nm}$ steps (Figure 3D, right). Thus, FIONA using Qdots provides a simple quantitative measurement for nano-scale tracking of proteins at the single molecular level.

\section{THREE-DIMENSIONAL SINGLE PARTICLE TRACKING WITH NANOMETER PRECISION USING Odots}

The original FIONA only measured movement on a spatial plane, but has since been expanded to three spatial dimensions. For this purpose, a three-dimensional (3D) image under a microscope is obtained by scanning the objective lens along the focal axis with an actuator (Watanabe and Higuchi, 2007; Wells et al., 2008). This scanning, however, decreases the temporal resolution of the tracking. To solve this problem, 3D tracking methods without the objective scanning have been developed (Genovesio et al., 2006; Holtzer et al., 2007; Watanabe et al., 2007; Ram et al., 2008, 2012; Wells et al., 2010; Jia et al., 2014). Multifocal planes microscopy uses the difference of distinct optical pathways to estimate the Z-position by obtaining 
simultaneously the fluorescence intensities of several focal images (Toprak et al., 2007; Watanabe et al., 2007; Dalgarno et al., 2010; Juette and Bewersdorf, 2010; Ram et al., 2012). Similarly, 3D tracking using a photon-limited double-helix response system with a spatial light modulator, which has two twisting lobes along the optical axis of the image, results in a single fluorescent probe appearing as two fluorescent spots from which the Z-position can be determined (Pavani et al., 2009; Lew et al., 2010).

One of the simplest 3D tracking methods intentionally generates astigmatism (Kao and Verkman, 1994; Holtzer et al., 2007; Izeddin et al., 2012). Here, a pair of convex and concave cylindrical lenses is inserted into the optical pathway before the detection device (Figure 4A) (Watanabe et al., 2013). These lenses generate different optical path lengths along the $\mathrm{X}$ - and $\mathrm{Y}$-axes, resulting in a measurable relationship between the Z-position of the particle and the ellipticity of the PSF (Figure 4B). To calculate the ellipticity in addition to the $2 \mathrm{D}$ position, the below approximation formula is used

$$
\begin{aligned}
f(x, y)= & I_{0} \cdot \exp \left\{-\frac{\left(x-x_{0}\right)^{2}}{2 \cdot \sigma_{x}^{2}}\right\} \cdot \exp \left\{-\frac{\left(y-y_{0}\right)^{2}}{2 \cdot \sigma_{y}^{2}}\right\} \\
& +C_{0}+C_{1} x+C_{2} y
\end{aligned}
$$

where $\sigma_{\mathrm{x}}$ and $\sigma_{\mathrm{y}}$ are the radial standard deviations of the Gaussian function along the $\mathrm{X}$ - and Y-axes respectively. The ellipticity is defined as the ratio of the full width at half maximum (FWHM) of the 2D Gaussian in the X- and Y- axes due to the different focal lengths (Figure 4C). Changing the distance between the convex and concave cylindrical lenses permits astigmatism for optimal tracking resolution (Figure 4D). When the detection device received 15,000 photons from a fluorescent probe, we achieved $3 \mathrm{D}$ tracking with precisions of $2 \mathrm{~nm}$ in the $\mathrm{X}$ and $\mathrm{Y}$-axes and $5 \mathrm{~nm}$ along the $\mathrm{Z}$-axis (Figure 4E). However, a reliable range was limited between a field view of -800 and $800 \mathrm{~nm}$ (Figure 4D, lower and Figure 4E). This drawback is common in many 3D tracking methods. A new 3D tracking method based on Airy beams, however, overcomes this problem. Here, a diffraction free self-bending PSF is applied to a two-channeled detection system (Jia et al., 2014), and the Z-position is translated to the distance difference of the two X-positions of the two channels. This method elongates the dynamic range of $3 \mathrm{D}$ tracking to $3 \mu \mathrm{m}$. Regardless of the $3 \mathrm{D}$ tracking method, the key is to extract $\mathrm{Z}$ information from the XY projection.

\section{FOUR-DIMENSIONAL SINGLE PARTICLE USING POLARIZED Qdots}

As significant as acquiring the third spatial dimension is, $3 \mathrm{D}$ single particle tracking ignores any rotational movement made by the protein. To acquire the orientation, fluorescence anisotropy can be used, because the fluorescence emissions are of unequal intensities along the $\mathrm{P}$ and $\mathrm{S}$ polar axes ( $\mathrm{P}$ - and $\mathrm{S}$-polarization), which are defined by the polarizing beam-splitter, as described below (Werver, 1953; Albrecht, 1961; Harms et al., 1999). Anisotropy is defined as $(I \mathrm{p}-I \mathrm{~s}) /(I \mathrm{p}+I \mathrm{~s})$, where $I \mathrm{p}$ and $I$ s are the intensities in P- and S-polarization, respectively (Harms et al., 1999). Anisotropy measurements have successfully tracked the rotatory dynamics of single protein molecules in vitro (Sase et al.,

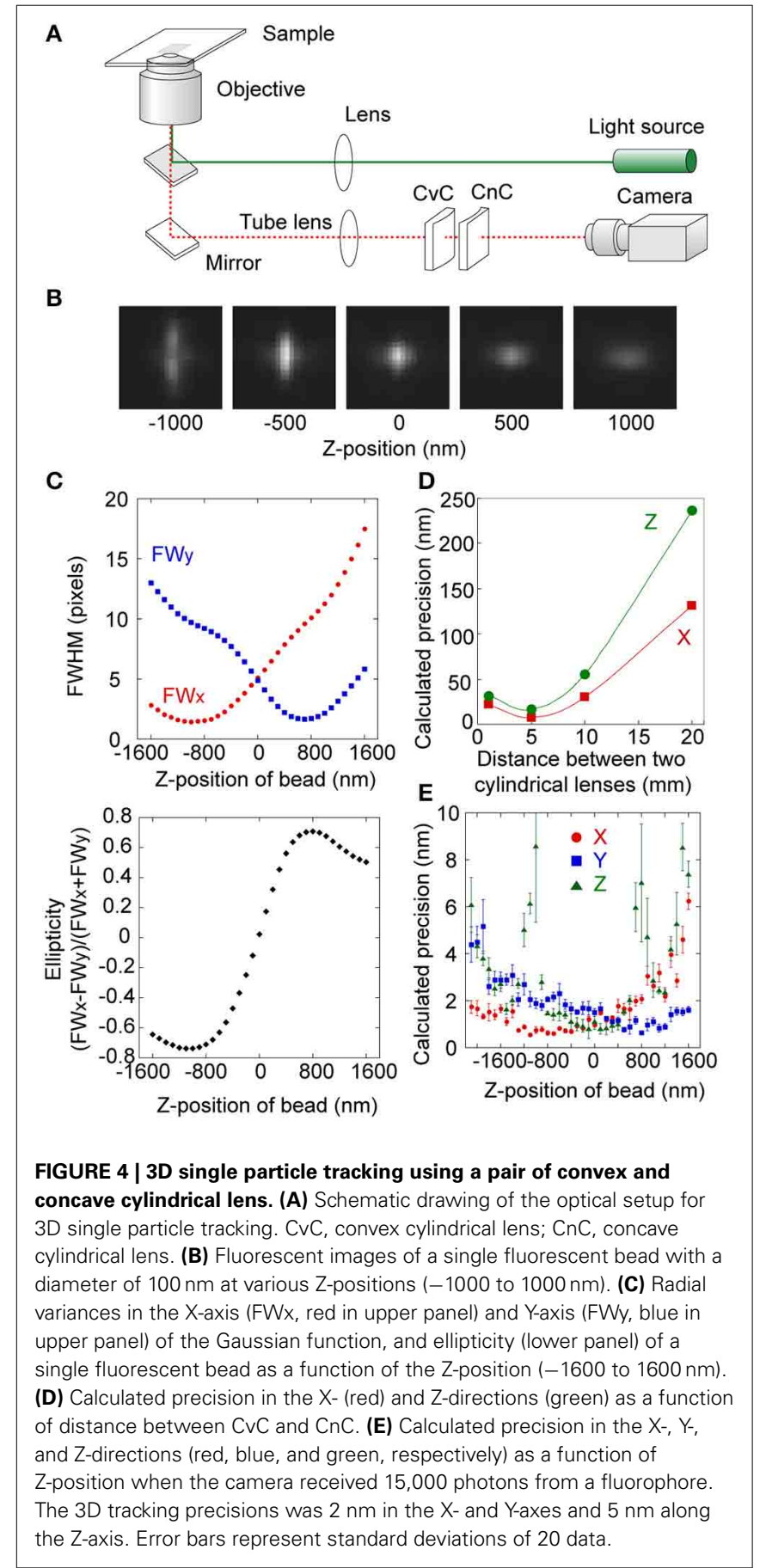

1997; Forkey et al., 2003) and in cells (Mizuno et al., 2011). The fluorescence anisotropy of a Qdot depends on the aspect ratio of its shape (Peng et al., 2000; Hu et al., 2001; Deka et al., 2009). Taking advantage of this property, a highly polarized rod-shaped Qdot (Qrod) can be synthesized by elongating the CdS shell along one-axis of the CdSe core (Figure 5A) (Peng et al., 2000; Hu et al., 2001). The anisotropy changes in Qrod fluorescence can be described as a sine function (Figures 5B,C) and the angular position by the arcsine function. The tracking precision of the 


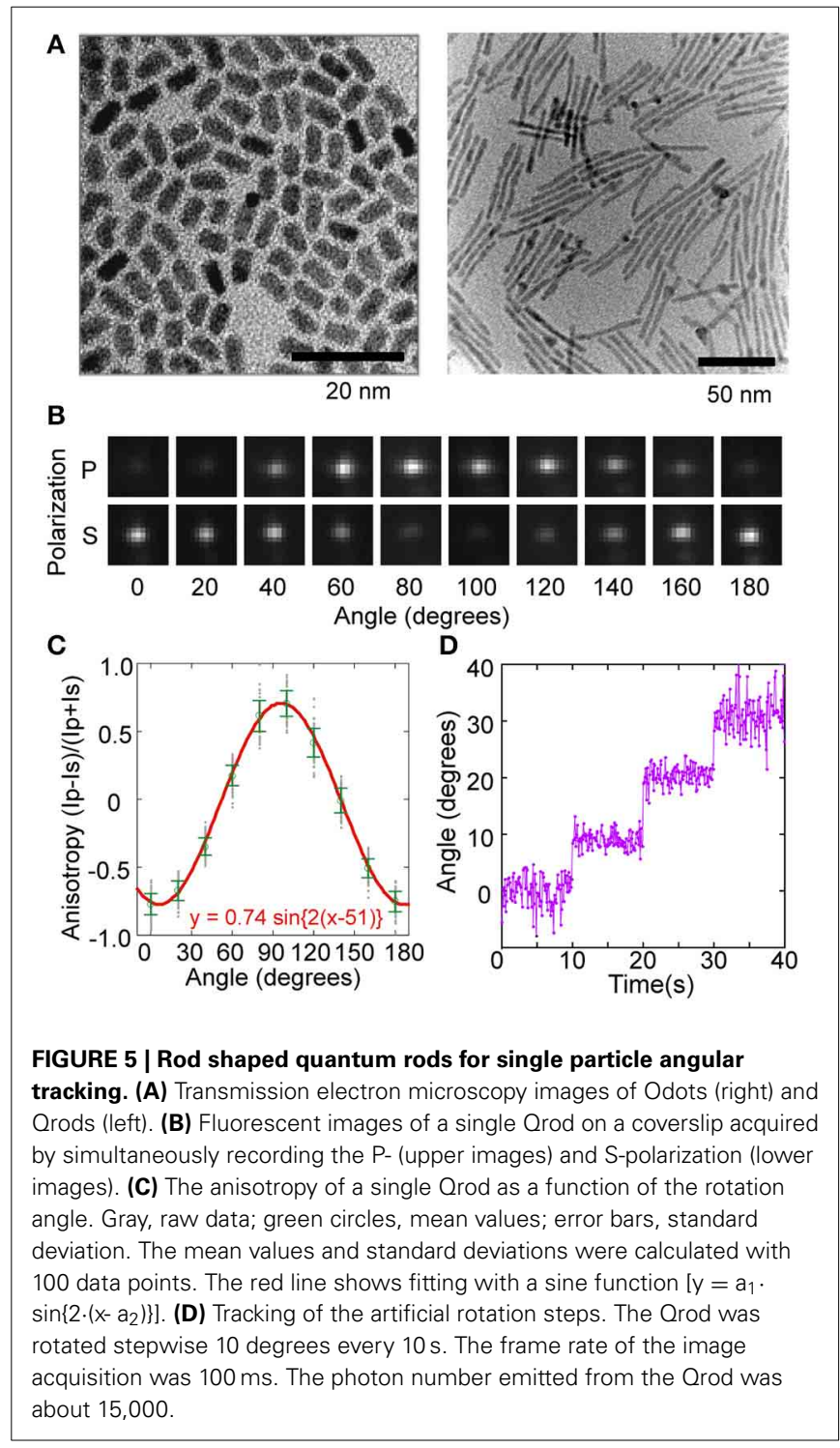

orientation was about $1 \sim 2^{\circ}$ when the photon number from a Qrod was 15,000 (Figure 8D). By utilizing this anisotropy technique, a fourth dimension, the angular $(\theta)$ component, could be added to the orthogonal 3 coordinate axes described by single particle tracking.

In our $4 \mathrm{D}$ tracking system, a polarizing beam splitter is set before the cylindrical lens pair in the 3D tracking optics to divide the fluorescent image into S- and P- polar channels (Figure 6A) (Watanabe et al., 2013). For 3D tracking, the P- and S-polarized images are summed before calculating the $3 \mathrm{D}$ position. A small gap is generated if the two channels are not completely overlapped, leading to an asymmetrical relationship between the respective FWHM values of the X- and Y-axes (Figure 6B). The $3 \mathrm{D}$ position can be determined by fitting the merged PSF with a 2D Gaussian function, as mentioned above, and the orientation can be determined by the ratio of the intensities of the $\mathrm{S}$ - and P-polarized images. Thus, $\mathrm{X}, \mathrm{Y}, \mathrm{Z}$, and $\theta$ are simultaneously obtained with an acquired image. In our case, when the number of photons from a single Qrod was about 10,000 and the $\mathrm{Z}$-position was near zero, the calculated precisions for the $\mathrm{X}, \mathrm{Y}, \mathrm{Z}$, and $\theta$-positions were at maximum $5,7,9 \mathrm{~nm}$ and $1^{\circ}$, respectively (Figure 6C).

We used 4D tracking to observe the movement of a membrane protein conjugated with a Qrod via antibody affinity (Figures 6D-F) (Watanabe et al., 2013). Isolated Qrods moving on the membrane were identified under a fluorescence microscope (Figure 6E). The different intensities in the P- and Spolarized images indicated that the Qrod was inclined against the optical axis (Figure 6F, upper panels). One circular and one elliptical spot indicated that the two Qrods were at distinct Z-positions (Figure 6F, lower panels). One Qrod showed a half-moon like motion in the $\mathrm{X}$ - and Y-axes, which was accompanied by highly fluctuating movements along the $\mathrm{Z}$-axis and fast rotational motion before endocytosis (Figure 6G). This observation suggests that this protein's lateral diffusion was constrained by the membrane undercoat, but that it could rotate freely along the plasma membrane. In the cytoplasm, a membrane protein seemed to be moving along tracks, most likely microtubules, in three-dimensions and slowly rotated helically (Figure 6H).

Another 4D tracking method was developed to obtain $\mathrm{X}, \mathrm{Y}$, $\theta$, and $\varphi$ coordinates, the last of which provides information on the out-of-plane tilt angle (Ohmachi et al., 2012). In this method, single Qrods are imaged as four crowded fluorescent spots by dividing the beam path using a beam splitter and two Wollaston prisms. Otherwise, the orientation of the individual fluorescent probe can be directly estimated using the dipole emission patterns of a defocused image (Bartko and Dickson, 1999a,b; Fourkas, 2001; Böhmer and Enderlein, 2003; Lieb et al., 2004), an approach that was successfully applied to the $4 \mathrm{D}$ tracking of a motor protein (Toprak et al., 2006). The combination of the Wollaston prism method with defocusing could achieve comprehensive tracking of all rotatory and translational movements of a biomolecule in a living cell.

\section{SUPER-RESOLUTION USING BLINKING OF Odots}

Super-resolution microscopy describes the resolution of two objects closer than the diffraction limit of light (Schermelleh et al., 2010; Galbraith and Galbraith, 2011; Leung and Chou, 2011). It can be classified into two main categories. The first is based on the photo-transition of a fluorescent probe between its radiative and non-radiative states in order to confine the fluorescence emission into a sub-diffraction-limit sized volume. This approach is known as RESOLFT (REversible Saturable OpticaL Fluorescence Transitions) and was first proposed and demonstrated by STED (STimulated Emission Depletion), which exploits the stimulated emission phenomenon of a fluorescent dye (Hell and Wichmann, 1994; Klar and Hell, 1999). RESOLFT can also be realized by other photoreactions, including those from a ground-state transition phenomenon (GSD: Ground State Depletion) (Hell and Kroug, 1995; Bretschneider et al., 2007), the saturation of fluorescence excitation (SAX: SAturated eXcitation) (Fujita et al., 2007), or from reversibly photoswitchable fluorescent proteins (Hofmann et al., 2005). RESOLFT can also be combined with structured illumination microscopy (SIM) (Heintzmann and Cremer, 1999; 

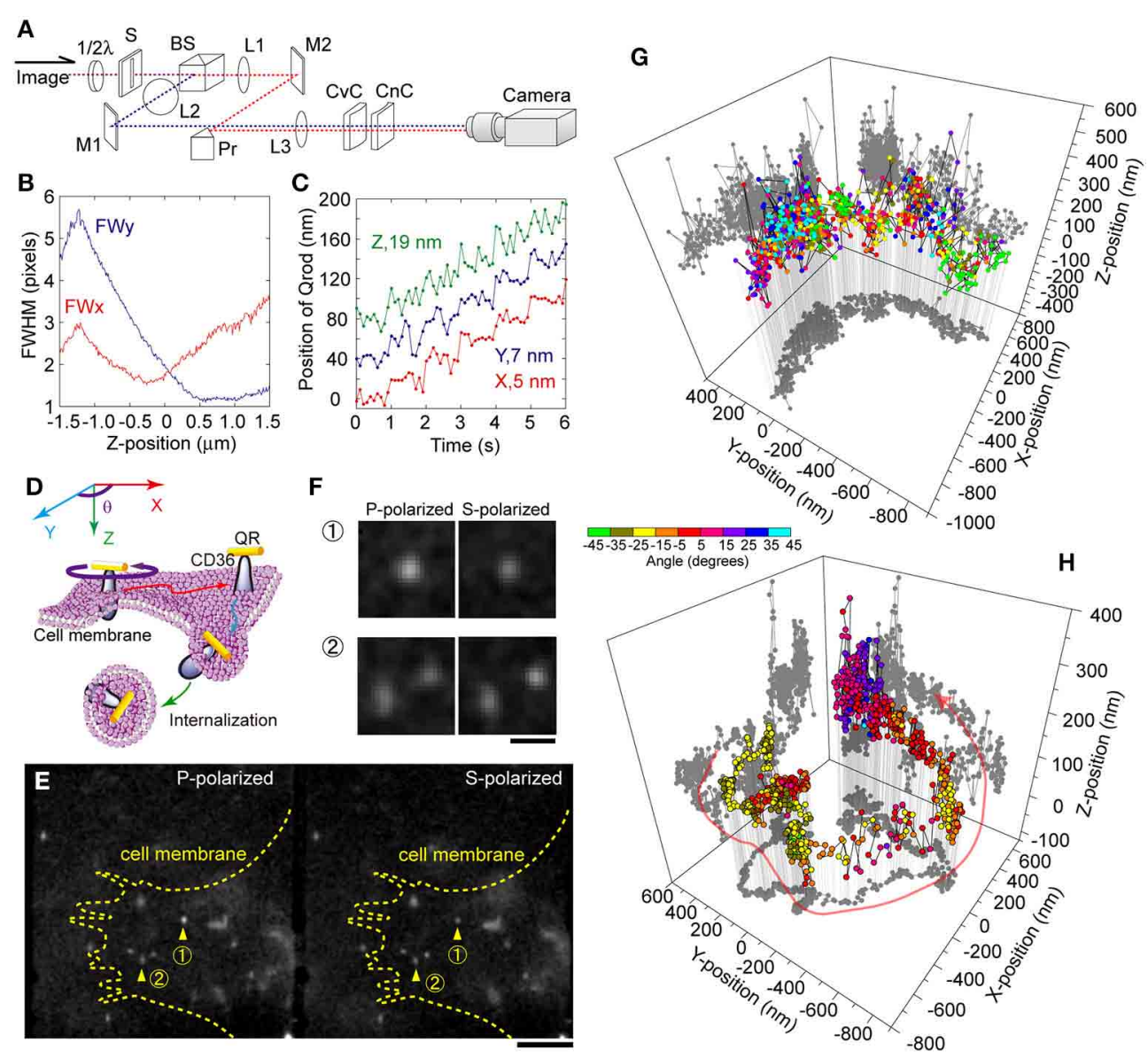

FIGURE 6 | 4D single particle tracking using Orod anisotropy. (A)

Schematic drawing of the optical setup for simultaneous $3 \mathrm{D}$ and angular single particle tracking. 1/2 $\lambda, 1 / 2$-wave plate; $S$, slit; $B S$, beam splitter; $L$, lens; $\mathrm{M}$, mirror; Pr, prism; CvC, convex cylindrical lens; and $\mathrm{CnC}$, concave cylindrical lens. (B) FWHM values of the merged images of $\mathrm{S}$ - and $\mathrm{P}$ - polarized images as a function of the Z-position along the $\mathrm{X}$ - (red line) and Y-axes (blue line). (C) Tracking $20 \mathrm{~nm}$ steps when the Z-position of the Orod was near zero. A Orod fixed on a coverslip was moved at discrete $20 \mathrm{~nm}$ steps once every $1 \mathrm{~s}$ simultaneously along the X-(red), Y- (blue), and Z- axes (green). The frame rate of the image acquisition was $100 \mathrm{~ms}$. Standard deviations of the tracking

for $10 \mathrm{~s}$ (excluding the stepping moments) were 5, 7, and $19 \mathrm{~nm}$ along the three respective axes. The photon number emitted from the Qrot was about 1500. (D) Schematic depiction of the internalization of Orod-labeled CD36, a membrane protein, from the cell membrane to the cytoplasm. (E) Fluorescent images of Qrods bound to membrane proteins in a living cell and simultaneously recorded in P- (left panel) and S-polarization (right panel). Arrowheads indicate two typical views that are enlarged in (F). Scale bar is $5 \mu \mathrm{m}$. (F) Enlarged images of the spots marked by arrowheads in (E). Scale bar, $1 \mu \mathrm{m}$. (G,H) Typical 4D traces of a single Qrod on the membrane $(\mathbf{G})$ and near the nucleus $\mathbf{( H )}$. The angle of the Orod is indicated by the color bar.

Gustafsson, 2000) to provide wide field imaging capability with superresolution (Heintzmann, 2003; Gustafsson, 2005).

The second category is based on the separate detection of individual single fluorescent probes in the time domain or spectra domain, and can be further decomposed into different concepts. One, known as SPDM (Spectral Precision Distance Microscopy), precisely localizes individual probes over the many frames of sequentially obtained images (Bornfleth et al., 1998; Lemmer et al., 2008). Stochastic optical reconstruction microscopy (STORM) (Rust et al., 2006) and fluorescence photoactivation localization microscopy (FPALM) (Betzig et al., 2006) are both SPDM-based techniques that utilize repeated activation-deactivation cycles of photoswitchable fluorophores such that the fluorescence spots on an obtained image are completely discrete.
Another method from the second category is blinking based superresolution (BBS). BBS relies on the randomness and nonGaussian property of blinking, which means stochastic processing can be used to localize individual fluorescent probes. The first report of BBS used independent component analysis, which is a computational method that decomposes a multivariate signal into independent non-Gaussian signals (Lidke et al., 2005). Other BBS-based techniques use the temporal high-order cumulant (super-resolution optical fluctuation imaging: SOFI) (Dertinger et al., 2009), the temporal high-order variance (Variance Imaging for Superresolution: VISion) (Watanabe et al., 2010), spatial covariance (spatial covariance reconstructive: SCORE) (Deng et al., 2014), or Bayesian statistics (Cox et al., 2011). A great advantage of SPDM and BBS is that they need only a relatively simple fluorescent microscope and no complicated optics. 
Qdots are the most compatible with BBS owing to their strong blinking phenomenon. Supposing that there are two adjoining Qdots independently and randomly fluctuating, the moment that one Qdot emits and the other does not is a stochastic event (Figure 7A). As an example, a solution to identifying the Qdot for SOFI and VISion is shown below (Dertinger et al., 2009). A fluorescent image of Qdots, $F(\boldsymbol{r}, t)$, is expressed by the convolution of a PSF, $U(\boldsymbol{r})$, of the optical system and the brightness, $\varepsilon_{k} s_{k}(t)$, where $\boldsymbol{r}_{k}, \varepsilon_{k}$, and $s_{k}(t)$ are the position and the time-invariant and -variant components of brightness of the $k$-th Qdot, respectively.

$$
F(r, t)=\sum_{k} U\left(\boldsymbol{r}-\boldsymbol{r}_{\mathbf{k}}\right) \cdot \varepsilon_{k} \cdot s_{k}(t)
$$

The second-order autocorrelation function, $G_{2}(\boldsymbol{r}, \tau)$, is then given by $F(\boldsymbol{r}, t)$ as follows,

$$
\begin{aligned}
G_{2}(r, \tau)= & \langle\delta F(\boldsymbol{r}, t+\tau) \cdot t F(\boldsymbol{r}, t)\rangle_{t} \\
= & \sum_{j, k} U\left(r-r_{j}\right) \cdot U\left(r-r_{k}\right) \cdot \varepsilon_{j} \cdot \varepsilon_{k} \cdot \\
& \left\langle\delta s_{j}(r, t+\tau) \cdot t s_{k}(r, t)\right\rangle_{t} \\
= & \sum_{k} U^{2}\left(r-r_{k}\right) \cdot \varepsilon_{k}^{2} \cdot\left\langle\delta s_{k}(r, t+\tau) \cdot t s_{k}(r, t)\right\rangle_{t}
\end{aligned}
$$

where $\langle\cdots\rangle_{\mathrm{t}}$ and $\delta(\cdot)$ denote a time-averaging operation and deviation from the time-average, respectively. Because of the independency of the fluorescence fluctuation of the two distinct Qdots $(k \neq j)$, the time average of their product is zero. For simple comparison of the raw image, $F(\boldsymbol{r}, t)$, and the auto-correlation image $G_{2}(\boldsymbol{r}, \tau)$, we here substitute 0 for the delay time, $\tau$, to reduce $G_{2}(\boldsymbol{r}, \tau)$ to $G_{2}(\boldsymbol{r}, 0)$.

$$
G_{2}(r, 0)=\sum_{k} U^{2}\left(r-r_{k}\right) \cdot \varepsilon_{k}^{2} \cdot\left\langle\delta s_{k}^{2}(t)\right\rangle_{t}
$$

This equation indicates that $\mathrm{G}_{2}(\mathbf{r}, \tau)$ is given by the convolution of $U^{2}(r)$ and the square of $\varepsilon_{\mathrm{k}} \mathrm{s}_{k}(\mathrm{t})$. Assuming that $U(\boldsymbol{r})$ is approximated by a Gaussian distribution, the spatial resolution of $G_{2}(\boldsymbol{r}, 0)$ is improved v2 times from $F(\boldsymbol{r}, t)$, but at the expense of temporal information, since the spatial resolution of the optical microscopic image is limited by the sharpness of the PSF (Figures 7B,C). The higher-order autocorrelation contains highspatial frequency information. However, because this is a moment value that contains cross-terms from the lower-order correlation contributions, the accrual spatial resolution for distinguishing two Qdots cannot be improved more than $\sqrt{ } 2$ times. It is therefore necessary to transform the $n$ th-order correlation into an $n$ th-order cumulant that consists only of terms containing the $n$th power of the PSF. While the higher order cumulant gives higher spatial resolution (Figure 7D), a huge number of images are still needed.

To decrease the required number of images, we developed a highly fluctuating Qdot in which the switching frequency between the on- and off-state was greatly increased by optimizing the shell thickness to promote more interaction between the CdSe-core and oxygen atoms in water (Figures $\mathbf{8 A , B}$ ). Though the quantum

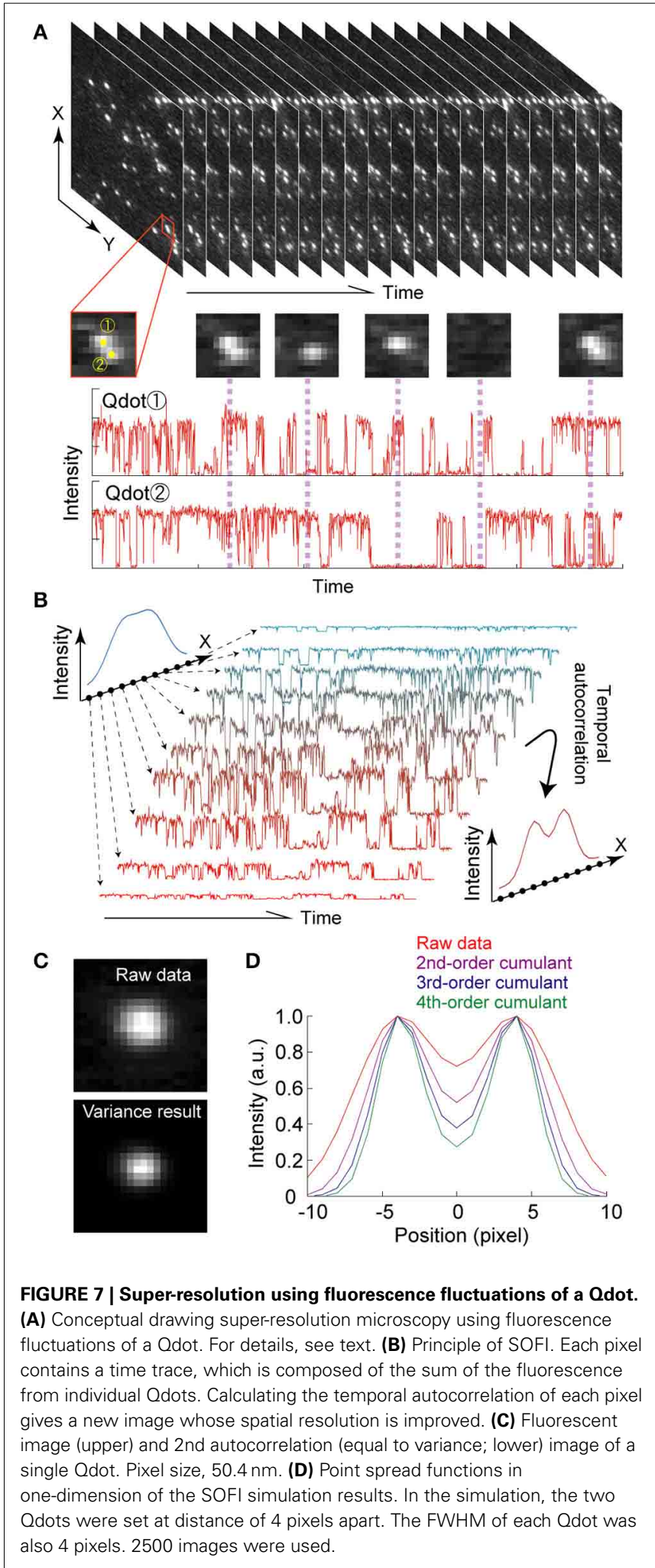

yield of this Qdot was less than that of standard Qdots, it still had sufficient intensity and stability when exposed to high power illumination, and no long off-state was observed (Figure 8C). Hence, we could easily obtain a super-resolved image by only 


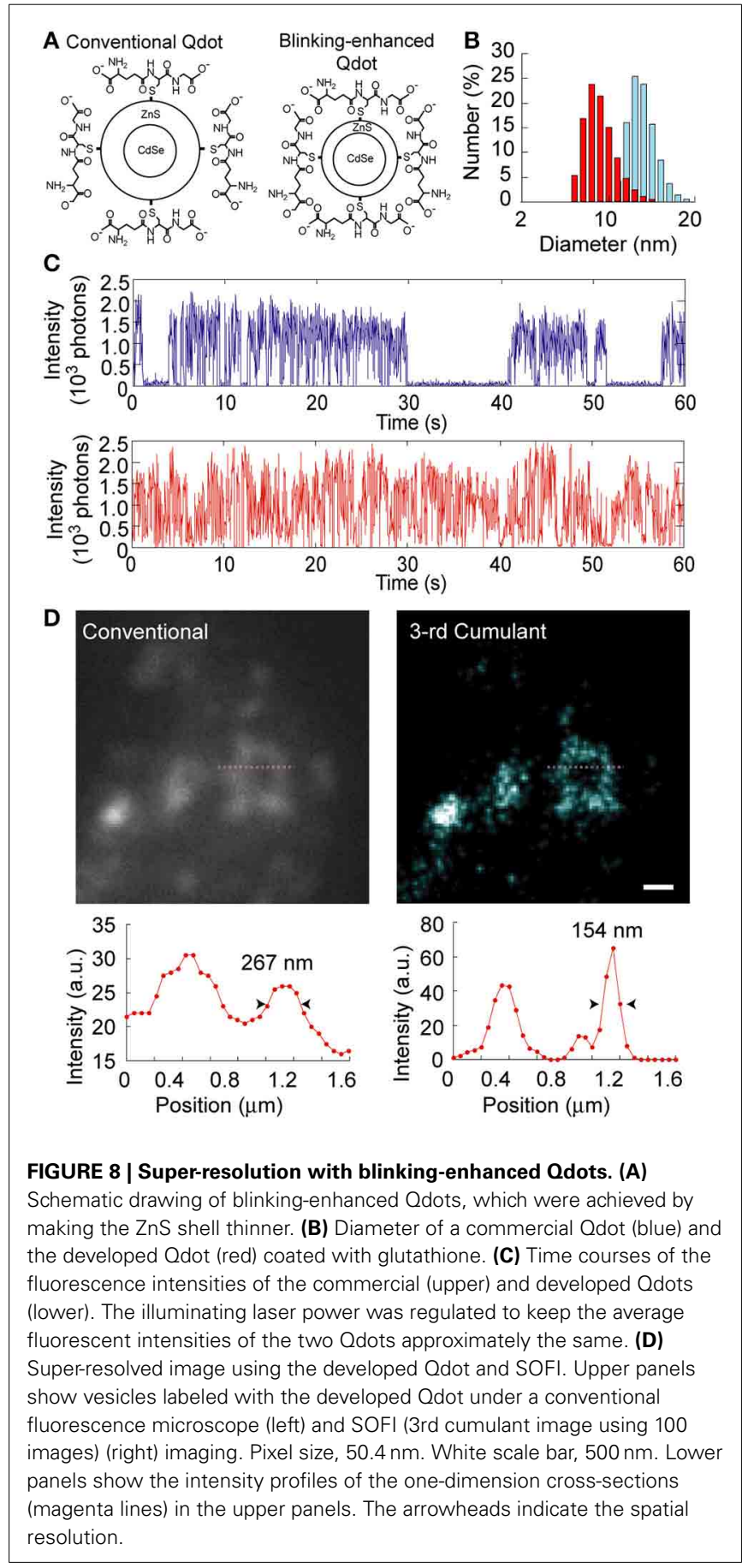

labeling the target protein and calculating the fluctuation of the blinking-enhanced Qdots (Figure 8D). In our case, the spatial resolution was improved from 267 to $154 \mathrm{~nm}$ using SOFI and only 100 images (Watanabe et al., 2010).

\section{CONCLUSION}

Conventional optical microscopy can quantitatively acquire 3D position and orientation information at the nano-scale from the shape of the PSF and the polarization characteristics of Qdots and Qrods. The amount of spatial information can be increased by analyzing the stochastic fluctuations of the fluorescence. Thus, the fluorescence of a probe attached to a molecule can reveal information about the molecular phenomena and/or state. Increasing the intensity, stability, and blinking of Qdots and its derivatives will make the acquisition of such information even more feasible.

Super-resolution microscopy and single particle tracking have made it possible to resolve and follow two objects closer than the diffraction limit of light. The result is quantitative information of the dynamics of biological phenomena at the nano-scale. Even more details of the dynamics can be acquired with the above technologies by using Qdots and their derivatives as probes for labeling the molecules of interest. The PSF and the polarization characteristics of the Qdots can be used to provide comprehensive information on both the position and orientation of the molecule of interest. Because this information can be extracted from the stochastic properties of the fluorescence, increasing the intensity, stability, and blinking of Qdots should provide even more quantitative details about the dynamics.

\section{ACKNOWLEDGMENT}

We gratefully acknowledge to Peter Karagiannis (Riken QBiC) for critical reading of this manuscript.

\section{REFERENCES}

Abbe, E. (1873). Contributions to the theory of the microscope and the microscopic perception. Arch. Mikr. Anat. 9, 413-468. doi: 10.1007/BF02956173

Adachi, K., Yasuda, R., Noji, H., Itoh, H., Harada, Y., Yoshida, M., et al. (2000). Stepping rotation of F1-ATPase visualized through angle-resolved single-fluorophore imaging. Proc. Natl. Acad. Sci. U.S.A. 97, 7243-7237. doi: 10.1073/pnas.120174297

Albrecht, A. (1961). Polarizations and assignments of transitions: the method of photoselection. J. Mol. Spectrosc. 6, 84-108. doi: 10.1016/0022-2852(61) 90234-X

Barroso, M. M. (2011). Quantum dots in cell biology. J. Histochem. Cytochem. 59, 237-251. doi: 10.1369/0022155411398487

Bartko, A. P., and Dickson, R. M. (1999a). Imaging three-dimensional single molecule orientations. J. Phys. Chem. B, 103, 11237-11241. doi: $10.1021 /$ jp993364q

Bartko, A. P., and Dickson, R. M. (1999b). Three-dimensional orientations of polymer-bound single molecules. J. Phys. Chem. B 103, 3053-3056. doi: 10.1021/jp9846330

Betzig, E., Patterso, G. H., Sougrat, R., Lindwasser, Q. W., Olenych, S., Bonifacino, J. S., et al. (2006). Imaging intracellular fluorescent proteins at nanometer resolution. Science 313, 1642-1645 doi: 10.1126/science.1127344

Böhmer, M., and Enderlein, J. (2003). Orientation imaging of single molecules by wide-field epifluorescence microscopy. J. Opt. Soc. Am. B 20, 554-559. doi: 10.1364/JOSAB.20.000554

Bornfleth, H., Satzler, K., Elis, R., and Cremer, C. (1998). High-precision distance measurements and volume-conserving segmentation of objects near and below the resolution limit in three-dimensional confocal fluorescence microscopy. J. Microsc. 189, 118-136. doi: 10.1046/j.1365-2818.1998.00276.x

Bretschneider, S., Eggeling, S., and Hell, S. W. (2007). Breaking the diffraction barrier in fluorescence microscopy by optical shelving. Phys. Rev. Lett. 98, 218103. doi: 10.1103/PhysRevLett.98.218103

Bruchez, M. Jr., Moronne, M., Gin, P., Weiss, S., and Alivisatos, A. P. (1998). Semiconductor nanocrystals as fluorescent biological labels. Science 281, 2013-2016. doi: 10.1126/science.281.5385.2016

Bruchez, M. P. (2011). Quantum dots find their stride in single molecule tracking. Curr. Opin. Chem. Biol. 15, 775-780. doi: 10.1016/j.cbpa.2011.10.011

Byers, R. J., and Hitchman, E. R. (2010). Quantum dots brighten biological imaging. Prog. Histochem. Cytochem. 45, 201-237. doi: 10.1016/j.proghi.2010.11.001

Cao, H., Chen, B., Squier, T. C., and Mayer, M. U. (2006). CrAsH: a biarsenical multi-use affinity probe with low non-specific fluorescence. Chem. Commun. 24, 2601-2603. doi: 10.1039/B602699K 
Chan, W. C. W., and Nie,. S. (1998). Quantum dot bioconjugates for ultrasensitive nonisotopic detection. Science 281, 2016-2018. doi: 10.1126/science.281.5385. 2016

Chang, Y. P., Pinaud, F., Antelman, J., and Weiss, S. (2008). Tracking bio-molecules in live cells using quantum dots. J. Biophotonics 1, 287-298. doi: 10.1002/jbio. 200810029

Cheezum, M. K., Walker, W. F., and Guilford, W. H. (2001). Quantitative comparison of algorithms for tracking single fluorescent particles. Biophys. J. 281, 2378-2388. doi: 10.1016/S0006-3495(01)75884-5

Chenouard, N., Smal, I., de Chaumont, F., Maška, M., Sbalzarini, I. F., Gong, Y., et al. (2014). Objective comparison of particle tracking methods. Nat. Methods. 11, 281-289. doi: 10.1038/nmeth.2808

Chien, F. C., Kuo, C. W., and Chen, P. (2011). Localization imaging using blinking quantum dots. Analyst 136, 1608-1613. doi: 10.1039/c0an00859a

Chinnathambi, S., Chen, S., Ganesan, S., and Hanagata, N. (2014). Silicon quantum dots for biological applications. Adv. Healthc. Mater. 3, 10-29. doi: 10.1002/adhm.201300157

Choi, H. S., and Frangioni, J. V. (2010). Nanoparticles for biomedical imaging: fundamentals of clinical translation. Mol. Imaging. 9, 291-310. doi: 10.2310/7290. 2010.00031

Clausen, M. P., and Lagerholm, B. C. (2011). The probe rules in single particle tracking. Curr. Protein Pept. Sci. 12, 699-713. doi: 10.2174/1389203117988 41672

Cox, S., Rosten, E., Monypenny, J., Jovanovic-Talisman, T., Burnette, D. T., Lippincott-Schwartz,.J., et al. (2011). Bayesian localization microscopy reveals nanoscale podosome dynamics. Nat. Methods 9, 195-200. doi: 10.1038/nmeth. 1812

Cupaioli, F. A., Zucca, F. A., Boraschi, D., and Zecca, L. (2014). Engineered nanoparticles. How brain friendly is this new guest? Prog Neurobiol. pii: S03010082(14)00051-3. doi: 10.1016/j.pneurobio.2014.05.002.

Dabbousi, B. O., Rodriguez-Viej, O. J., and Bawendi, M. G. (1997). (CdSe)ZnS core-shell Qdots: synthesis and characterization of a size series of highly luminescent nanocrystallites. J. Phys. Chem. B 101, 9463-9475. doi: 10.1021/ jp971091y

Dalgarno, P. A., Dalgarno, H. I., Putoud, A., Lambert, R., Paterson, L., Logan, D. C., et al. (2010). Multiplane imaging and three dimensional nanoscale particle tracking in biological microscopy. Opt. Express. 18, 877-884. doi: 10.1364/OE. 18.000877

Deka, S., Quarta, A., Lupo, M. G., Falqui, A., Boninelli, S., Giannini, C., et al. (2009). CdSe/CdS/ZnS double shell nanorods with high photoluminescence efficiency and their exploitation as biolabeling probes. J. Am. Chem. Soc. 131, 2948-2958. doi: 10.1021/ja808369e

Deng, Y., Sun, M., Lin, P. H., Ma, J., and Shaevitz, J. W. (2014). Spatial covariance reconstructive (SCORE) super-resolution fluorescence microscopy. PLoS ONE 9:e94807. doi: 10.1371/journal.pone.0094807

Dertinger, T., Colyer, R., Iyer, G., Weiss, S., and Enderlein, J. (2009). Fast, background-free, 3D super-resolution optical fluctuation imaging (SOFI). Proc. Natl. Acad. Sci. U.S.A. 106, 22287-22292. doi: 10.1073/pnas.0907866106

Deschout, H., Cella Zanacchi, F., Mlodzianoski, M., Diaspro, A., and Bewersdorf, J. (2014). Precisely and accurately localizing single emitters in fluorescence microscopy. Nat. Methods. 11, 253-266. doi: 10.1038/nmeth.2843

Drummen, G. P. (2012). Fluorescent probes and fluorescence (microscopy) techniques-illuminating biological and biomedical research. Molecules 17, 14067-11490. doi: 10.3390/molecules 171214067

Dubertret, B., Skourides, P., Norris, D. J., Noireaux, V., Brivanlou, A. H., and Libchaber, A. (2002). In vivo imaging of quantum dots encapsulated in phospholipid micelles. Science 98, 1759-1762. doi: 10.1126/science.1077194

Dubois, F., Mahler, B., Dubertret, B., Doris, E., and Mioskowski, C. (2007). A versatile strategy for quantum dot ligand exchange. J. Am. Chem. Soc. 129, 482-483. doi: $10.1021 /$ ja067742y

Ekimov, A. I., and Onushchenko, A. A. (1981). Quantum size effect in threedimensional microscopic semiconductor crystals. JETP Lett. 34, 345-349.

Ellinger, P. (1940). Fluorescence microscopy in biology. Biol. Rev. 15, 323-347. doi: 10.1111/j.1469-185X.1940.tb00761.x

Erathodiyil, N., and Ying, J. Y. (2011). Functionalization of inorganic nanoparticles for bioimaging applications. Acc. Chem. Res. 44, 925-935. doi: 10.1021/ ar2000327

Fomenko, V., and Nesbitt, D. J. (2008). Solution control of radiative and nonradiative lifetimes: a novel contribution to quantum dot blinking suppression. Nano Lett. 8, 287-293. doi: 10.1021/nl0726609
Forkey, J. N., Quinlan, M. E., Shaw, M. A., Corrie, J. E., and Goldman, Y. E. (2003). Three-dimensional structural dynamics of myosin V by single-molecule fluorescence polarization. Nature 422, 399-404. doi: 10.1038/nature01529

Fourkas, J. T. (2001). Rapid determination of the three-dimensional orientation of single molecules. Opt. Lett. 26, 211-213. doi: 10.1364/OL.26.000211

Fujita, K., Kobayashi, M., Kawano, S., Yamanaka, M., and Kawata, S. (2007). Highresolution confocal microscopy by saturated excitation of fluorescence. Phys Rev. Lett. 99:228105. doi: 10.1103/PhysRevLett.99.228105

Galbraith, C. G., and Galbraith, J. A. (2011). Super-resolution microscopy at a glance. J Cell Sci. 124(Pt 10), 1607-1611. doi: 10.1242/jcs.080085

Gao, X., Yang, L., Petros, J. A., Marshall, F. F., Simons, J. W., and Nie, S. (2005). In vivo molecular and cellular imaging with quantum dots. Curr. Opin. Biotechnol. 16, 63-72. doi: 10.1016/j.copbio.2004.11.003

Genovesio, A., Liedl, T., Emiliani, V., Parak, W. J., Coppey-Moisan, M., and OlivoMarin, J. C. (2006). Multiple particle tracking in 3-D+t microscopy: method and application to the tracking of endocytosed quantum dots. IEEE Trans. Image Process. 15, 1062-1070. doi: 10.1109/TIP.2006.872323

Gerion, D., Pinaud, F., Williams, S., Parak, W., Zanchet, D., Weiss, S., et al. (2001). Synthesis and properties of biocompatible water-soluble silica-coated CdSe/ZnS semiconductor quantum dots. J. Phys. Chem. B 105, 8861-8871 doi: 10.1021/jp0105488

Guo, W., Li, J. J., Wang, Y. A., Peng, X. (2003). Conjugation chemistry and bioapplications of semiconductor box nanocrystals prepared via dendrimer bridging. Chem. Mater. 15, 3125-3133. doi: 10.1021/cm034341y

Gustafsson, M. G. L. (2000). Surpassing the lateral resolution limit by a factor of two using structured illumination microscopy. J. Microsc. 198, 82-87 doi: 10.1046/j.1365-2818.2000.00710.x

Gustafsson, M. G. L. (2005). Nonlinear structured-illumination microscopy: Widefield fluorescence imaging with theoretically unlimited resolution. Proc. Natl. Acad. Sci. U.S.A. 102, 13081-13086. doi: 10.1073/pnas.0406877102

Harms, G. S., Sonnleitner, M., Schütz, G. J., Gruber, H. J., and Schmidt, T. (1999). Single-molecule anisotropy imaging. Biophys. J. 77, 2864-2870. doi: 10.1016/S0006-3495(99)77118-3

Heintzmann, R. (2003). Saturated patterned excitation microscopy with twodimensional excitation patterns. Micron 34, 283-291. doi: 10.1016/S09684328(03)00053-2

Heintzmann, R., and Cremer, C. (1999). "Laterally modulated excitation microscopy: improvement of resolution by using a diffraction grating," in Proceedings for the SPIE Vol. 3568, Optical Biopsies and Microscopic Techniques III, (Stockholm), 185. doi: 10.1117/12.336833

Hell, S. W., and Kroug, M. (1995). Ground-state-depletion fluorescence microscopy: A concept for breaking the diffraction resolution limit. Appl. Phys. B 60, 495-497. doi: 10.1007/BF01081333

Hell, S. W., and Wichmann, J. (1994). Breaking the diffraction resolution limit by stimulated emission: stimulated-emission-depletion fluorescence microscopy. Opt. Lett. 19, 780-782. doi: 10.1364/OL.19.000780

Hoffman, M. T., Sheung, J., and Selvin, P. R. (2011). Fluorescence imaging with one nanometer accuracy: in vitro and in vivo studies of molecular motors. Methods Mol. Biol. 778, 33-56. doi: 10.1007/978-1-61779-261-8_4

Hofmann, M., Eggeling, C., Jakobs, S., and Hell, S. W. (2005). Breaking the diffraction barrier in fluorescence microscopy at low light intensities by using reversibly photoswitchable proteins. Proc. Natl. Acad. Sci. U.S.A.102, 17565-17569. doi: 10.1073/pnas.0506010102

Hohng, S., and Ha, T. (2004). Near-complete suppression of quantum dot blinking in ambient conditions. J. Am. Chem. Soc. 126, 1324-1325. doi: 10.1021/ja039686w

Holtzer, L., Meckel, T., and Schmidt, T. (2007). Nanometric three-dimensional tracking of individual quantum dots in cells. Appl. Phys. Lett. 90, 053902 doi: 10.1063/1.2437066

Hoyer, P., Staudt, T., Engelhardt, J., and Hell, S. W. (2011). Quantum dot blueing and blinking enables fluorescence nanoscopy. Nano Lett. 11, 245-250. doi: $10.1021 / \mathrm{nl} 103639 \mathrm{f}$

Hu, J., Li, L. S., Yang, W., Manna, L., Wang, L. W., and Alivisatos, A. P. (2001). Linearly polarized emission from colloidal semiconductor quantum rods. Science. 292, 2060-2063. doi: 10.1126/science.1060810

Huang, Z. L., Zhu, H., Long, F., Ma, H., Qin, L., Liu, Y., et al. (2011). Localizationbased super-resolution microscopy with an sCMOS camera. Opt. Express. 19, 19156-19168. doi: 10.1364/OE.19.019156

Izeddin, I., El Beheiry, M., Andilla, J., Ciepielewski, D., Darzacq, X., and Dahan, M. (2012). PSF shaping using adaptive optics for three-dimensional 
single-molecule super-resolution imaging and tracking. Opt. Express. 20, 4957-4967. doi: 10.1364/OE.20.004957

Jia, S., Vaughan, V. C., and Zhuang, Z. (2014). Isotropic three-dimensional superresolution imaging with a self-bending point spread function. Nat. Photonics 8 , 302-306. doi: 10.1038/nphoton.2014.13

Jiang, W., Mardyani, S., Fischer, H., and Chan, W. C. W. (2006). Design and characterization of lysine cross-linked mercapto-acid biocompatible quantum dots. Chem. Mater. 18, 872-878. doi: 10.1021/cm051393+

Jin, T., Fujii, F., Komai, Y., Seki, J., Seiyama, A., and Yoshioka, Y. (2008). Preparation and characterization of highly fluorescent, glutathione-coated near infrared quantum dots for in vivo fluorescence imaging. Int. J. Mol. Sci. 9, 2044-2061. doi: 10.3390/ijms 9102044

Juette, M. F., and Bewersdorf, J. (2010). Three-dimensional tracking of single fluorescent particles with submillisecond temporal resolution. Nano Lett. 10, 4657-4663. doi: 10.1021/nl1028792.

Kairdolf, B. A., Smith, A. M., Stokes, T. H., Wang, M. D., Young, A. N., and Nie, S. (2013). Semiconductor quantum dots for bioimaging and biodiagnostic applications. Annu. Rev. Anal. Chem. 6, 143-162. doi: 10.1146/annurev-anchem060908-155136

Kao, H. P., and Verkman, A. S. (1994). Tracking of single fluorescent particles in three dimensions: use of cylindrical optics to encode particle position. Biophys. J. 67, 1291-1300. doi: 10.1016/S0006-3495(94)80601-0

Kim, S. W., Kim, S., Tracy, J. B., Jasanoff, A., and Bawendi, M. G. (2005). Phosphine oxide polymer for water-soluble nanoparticles. J. Am. Chem. Soc. 127, 4556-4557. doi: 10.1021/ja043577f

Klar, T. A., and Hell, S. W. (1999). Subdiffraction resolution in far-field fluorescence microscopy. Opt. Lett. 24, 954-956. doi: 10.1364/OL.24.000954

Kloepfer, J. A., Bradforth, S. E., and Nadeau, J. L. (2005). Photophysical properties of biologically compatible CdSe quantum dot structures. J. Phys. Chem. B 109, 9996-10003. doi: 10.1021/jp044581g

Ko, H. C., Yuan, C. T., and Tang, J. (2011). Probing and controlling fluorescence blinking of single semiconductor nanoparticles. Nano Rev. 2:5895. doi: 10.3402/ nano.v2i0.5895

Kubitscheck, U., Kückmann, O., Kues, T., and Peters, R. (2000). Imaging and tracking of single GFP molecules in solution. Biophys. J. 78, 2170-2179. doi: 10.1016/S0006-3495(00)76764-6

Kuno, M., Lee, J. K., Dabbousi, B. O., Mikulec, F. V., and Bawendi, M. G. (1997). The band edge luminescence of surface modified CdSe nanocrystallites: probing the luminescing state. J. Chem. Phys. 106, 9869. doi: 10.1063/1.473875

Kusumi, A., Nakada, C., Ritchie, K., Murase, K., Suzuki, K., Murakoshi, H., et al. (2005). Paradigm shift of the plasma membrane concept from the two-dimensional continuum fluid to the partitioned fluid: high-speed singlemolecule tracking of membrane molecules. Annu. Rev. Biophys. Biomol. Struct. 34, 351-378. doi: 10.1146/annurev.biophys.34.040204.144637

Lasne, D., Blab, G. A., Berciaud, S., Heine, M., Groc, L., Choquet, D., et al. (2006). Single nanoparticle photothermal tracking (SNaPT) of 5-nm gold beads in live cells. Biophys. J. 91, 4598-4604. doi: 10.1529/biophysj.106.089771

Lemmer, P., Gunkel, M., Baddeley, D., Kaufmann, R., Urich, A., Weiland, Y., et al. (2008). SPDM: light microscopy with single-molecule resolution at the nanoscale. Appl. Phys. B 93, 1-12. doi: 10.1007/s00340-008-3152-x

Leung, B. O., and Chou, K. C. (2011). Review of super-resolution fluorescence microscopy for biology. Appl. Spectrosc. 65, 967-980. doi: 10.1366/11-06398

Levenberg, K. (1944). A method for the solution of certain non-linear problems in least squares. Q. Appl. Math. 2, 164-168.

Lew, M. D., Thompson, M. A., Badieirostami, M., and Moerner, W. E. (2010). In vivo three-dimensional superresolution fluorescence tracking using a doublehelix point spread function. Proc. Soc. Photo Opt. Instrum. Eng. 7571, 75710Z. doi: $10.1117 / 12.842608$

Li, J., Wu, D., Miao, Z., and Zhang, Y. (2010). Preparation of quantum dot bioconjugates and their applications in bio-imaging. Curr. Pharm. Biotechnol. 11, 662-671. doi: 10.2174/138920110792246582

Lichtman, J. W., and Conchello, J. A. (2005). Fluorescence microscopy. Nat. Methods 2, 910-919. doi: 10.1038/nmeth817

Lidke, K., Rieger, B., Jovin, T., and Heintzmann, R. (2005). Superresolution by localization of quantum dots using blinking statistics. Opt. Express. 13, 7052-7062. doi: 10.1364/OPEX.13.007052

Lieb, M. A., Zavislan, J. M., and Novotny, L. (2004). Single-molecule orientations determined by direct emission pattern imaging. J. Opt. Soc. Am. B 21, 1210-1215. doi: 10.1364/JOSAB.21.001210
Long, F., Zeng, S., and Huang, Z. L. (2012). Localization-based super-resolution microscopy with an sCMOS camera part II: experimental methodology for comparing sCMOS with EMCCD cameras. Opt. Express. 20, 17741-17759. doi: 10.1364/OE.20.017741

Ma, H., Kawai, H., Toda, E., Zeng, S., and Huang, Z. L. (2013). Localizationbased super-resolution microscopy with an sCMOS camera part III: camera embedded data processing significantly reduces the challenges of massive data handling. Opt. Lett. 38, 1769-1771. doi: 10.1364/OL.38.001769

Mandal, A., and Tamai, N. (2011). Suppressed blinking behavior of thioglycolic acid capped CdTe quantum dot by amine functionalization. Appl. Phys. Lett. 99, 263111. doi: $10.1063 / 1.3671075$

Miyawaki, A. (2013). Fluorescence imaging in the last two decades. Microscopy (Oxf.). 62, 63-68. doi: 10.1093/jmicro/dfs 130

Mizuno, H., Higashida, C., Yuan, Y., Ishizaki, T., Narumiya, S., and Watanabe, N. (2011). Rotational movement of the formin mDial along the double helical strand of an actin filament. Science 331, 80-83. doi: 10.1126/science.1197692

Nann, T. (2005). Phase-transfer of CdSe@ZnS quantum dots using amphiphilic hyperbranched polyethylenimine. Chem. Commun. 7, 1735-1736. doi: $10.1039 / \mathrm{b} 414807 \mathrm{j}$

Nifosí, R., Amat, P., and Tozzini, V. (2007). Variation of spectral, structural, and vibrational properties within the intrinsically fluorescent proteins family: a density functional study. J. Comput. Chem. 28, 2366-2377. doi: 10.1002/jcc.20764

Nirmal, M., Dabbousi, B. O., and Brus, L. E. (1996). Fluorescence intermittency in single cadmium selenide nanocrystals. Nature 383, 802-804. doi: $10.1038 / 383802 \mathrm{a} 0$

Nishikawa, S., Arimoto, I., Ikezaki, K., Sugawa, M., Ueno, H., Komori, T., et al. (2010). Switch between large hand-over-hand and small inchworm-like steps in myosin VI. Cell 42, 879-888. doi: 10.1016/j.cell.2010.08.033

Ohmachi, M., Komori, Y., Iwane, A. H., Fujii, F., Jin, T., and Yanagida, T. (2012). Fluorescence microscopy for simultaneous observation of $3 \mathrm{D}$ orientation and movement and its application to quantum rod-tagged myosin V. Proc. Natl. Acad. Sci. U.S.A. 109, 5294-5298. doi: 10.1073/pnas.1118472109

Palaniappan, K., Hackney, S. A., and Liu, J. (2004). Supramolecular control of complexation-induced fluorescence change of water-soluble, betacyclodextrin-modified CdS quantum dots. Chem. Commun., 23, 2704-2705. doi: 10.1039/B409075F

Palaniappan, K.,Xue, C., Arumugam, G., Hackney, S. A., and Liu, J. (2006). Watersoluble, cyclodextrin-modified CdSe-CdS core-shell structured quantum dots. Chem. Mater. 18, 1275-1280. doi: 10.1021/cm051602q

Park, H., Toprak, E., and Selvin, P. R. (2007). Single-molecule fluorescence to study molecular motors. Q. Rev. Biophys. 40, 87-111. doi: 0.1017/S0033583507004611

Pavani, S. R., Thompson, M. A., Biteen, J. S., Lord, S. J., Liu, N., Twieg, R. J., et al. (2009). Three-dimensional, single-molecule fluorescence imaging beyond the diffraction limit by using a double-helix point spread function. Proc. Natl. Acad. Sci. U.S.A. 106, 2995-2999. doi: 10.1073/pnas.0900245106

Peng, X., Manna, L., Yang, W., Wickham, J., Scher, E., Kadavanich, A., et al. (2000). Shape control of CdSe nanocrystals. Nature 404, 59-61. doi: 10.1038/35003535

Peter, S., Harter, K., and Schleifenbaum, F. (2014). Fluorescence microscopy. Methods Mol. Biol. 1062, 429-452. doi: 10.1007/978-1-62703-580-4_23

Petryayeva, E., Algar, W. R., and Medintz, I. L. (2013). Quantum dots in bioanalysis: a review of applications across various platforms for fluorescence spectroscopy and imaging. Appl. Spectrosc. 67, 215-252. doi: 10.1366/ 12-06948

Pierobon, P., and Cappello, G. (2012). Quantum dots to tail single biomolecules inside living cells. Adv. Drug Deliv. Rev. 64, 167-178. doi: 10.1016/j.addr.2011.06.004

Pilla, V., Munin, E., Dantas, N. O., Silva, A. C. A., and Andrade, A. A. (2012). "Photothermal spectroscopic characterization in CdSe/ZnS and CdSe/CdS quantum dots: a review and new applications," in Quantum Dots - A Variety of New Applications, ed A. Al-Ahmadi (InTech), 3-22. doi: 10.5772/2645

Pinaud, F., King, D., Moore, H. P., and Weiss, S. (2004). Bioactivation and cell targeting of semiconductor CdSe/ZnS nanocrystals with phytochelatin-related peptides. J. Am. Chem. Soc. 126, 6115-6123. doi: 10.1021/ja031691c

Ram, S., Kim, D., Ober, R. J., and Ward, E. S. (2012). 3D single molecule tracking with multifocal plane microscopy reveals rapid intercellular transferrin transport at epithelial cell barriers. Biophys. J. 103, 1594-1603. doi: 10.1016/j.bpj.2012.08.054

Ram, S., Prabhat, P., Chao, J., Ward, E. S., and Ober, R. J. (2008). High accuracy $3 \mathrm{D}$ quantum dot tracking with multifocal plane microscopy for the study 
of fast intracellular dynamics in live cells. Biophys. J. 95, 6025-6043. doi: 10.1529/biophysj.108.140392

Ritchie, K., and Kusumi, A. (2003). Single-particle tracking image microscopy. Meth. Enzymol. 360, 618-634. doi: 10.1016/S0076-6879(03)60131-X

Rossetti, R., Nakahara, S., and Brus, L. E. (1980). Quantum size effects in the redox potentials, resonance Raman spectra, and electronic spectra of CdS crystallites in aqueous solution. J. Chem. Phys. 79, 1086-1088 doi: 10.1063/1.445834

Ruedas-Rama, M. J., Walters, J. D., Orte, A., and Hall, E. A. (2012). Fluorescent nanoparticles for intracellular sensing: a review. Anal. Chim. Acta 751, 1-23. doi: 10.1016/j.aca.2012.09.025

Rust, M. J., Bate, M., and Zhuang, X. (2006). Sub-diffraction-limit imaging by stochastic optical reconstruction microscopy (STORM). Nat. Methods 3, 793-796 doi: 10.1038/nmeth929

Ruthardt, N., Lamb, D. C., and Bräuchle, C. (2011). Single-particle tracking as a quantitative microscopy-based approach to unravel cell entry mechanisms of viruses and pharmaceutical nanoparticles. Mol. Ther. 19, 1199-1211. doi: $10.1038 / \mathrm{mt} .2011 .102$

Saadeh, Y., Leung, T., Vyas, A., Chaturvedi, L. S., Perumal, O., and Vyas, D. (2014). Applications of nanomedicine in breast cancer detection, imaging, and therapy. J. Nanosci. Nanotechnol. 14, 913-923. doi: 10.1166/jnn.2014.8755

Sambongi, Y., Iko, Y., Tanabe, M., Omote, H., Iwamoto-Kihara, A., Ueda, I., et al. (1999). Mechanical rotation of the $\mathrm{c}$ subunit oligomer in ATP synthase (F0F1): direct observation. Science 286, 1722-1724. doi: 10.1126/science.286. 5445.1722

Sase, I., Miyata, H., Ishiwata, S., and Kinosita, K. Jr. (1997). Axial rotation of sliding actin filaments revealed by single-fluorophore imaging. Proc. Natl. Acad. Sci. U.S.A. 94, 5646-5650. doi: 10.1073/pnas.94.11.5646

Saxton, M. J. (2008). Single-particle tracking: connecting the dots. Nat. Methods 5, 671-672. doi: 10.1038/nmeth0808-671

Saxton, M. J. (2009). "Single particle tracking," in Fundamental Concepts of Biophysics, ed T. Jue (New York, NY: Humana Press), 147-169. doi: 10.1007/ 978-1-59745-397-4_6

Schermelleh, L., Heintzmann, R., and Leonhardt, H. (2010). A guide to super-resolution fluorescence microscopy. J. Cell Biol. 190, 165-175. doi: 10.1083/jcb.201002018

Schlegel, G., Bohnenberger, J., Potapova, I., and Mews, A. (2002). Fluorescence decay time of single semiconductor nanocrystals. Phys. Rev. Lett. 88, 137401. doi: 10.1103/PhysRevLett.112.068103

Shimomura, O., and Johnson, F. H. (1692). Extraction, purification and properties of aequorin, a bioluminescent protein from the luminous hydromedusan. Aequorea. J. Cell. Comp. Physiol. 59, 223-239. doi: 10.1002/jcp.1030590302

Small, A., and Stahlheber, S. (2014). Fluorophore localization algorithms for superresolution microscopy. Nat. Methods. 11, 267-279. doi: 10.1038/nmeth.2844

Terai, T., and Nagano, T. (2013). Small-molecule fluorophores and fluorescent probes for bioimaging. Pflugers Arch. 465, 347-359. doi: 10.1007/s00424-0131234-z

Thompson, R. E., Larson, D. R., and Webb, W. W. (2002). Precise nanometer localization analysis for individual fluorescent probes. Biophys. J. 82, 2775-2783. doi: 10.1016/S0006-3495(02)75618-X

Tiwari, D. K., Tanaka, S., Inouye, Y., Yoshizawa, K., Watanabe, T. M., and Jin, T. (2009). Synthesis and characterization of anti-HER2 antibody conjugated CdSe/CdZnS quantum dots for fluorescence imaging of breast cancer cells. Sensors 9, 9332-9364. doi: 10.3390/s91109332

Tomczak, N., Liu, R., and Vancso, J. G. (2013). Polymer-coated quantum dots. Nanoscale 5, 12018-12032. doi: 10.1039/c3nr03949h

Toprak, E., Balci, H., Blehm, B. H., and Selvin, P. R. (2007). Threedimensional particle tracking via bifocal imaging. Nano Lett. 7, 2043-2045. doi: $10.1021 / \mathrm{nl} 0709120$

Toprak, E., Enderlein, J., Syed, S., McKinney, S. A., Petschek, R. G., Ha, T., et al. (2006). Defocused orientation and position imaging (DOPI) of myosin V. Proc. Natl. Acad. Sci. U.S.A. 103, 6495-6499. doi: 10.1073/pnas.0507134103

Toprak, E., and Selvin, P. R. (2007). New fluorescent tools for watching nanometerscale conformational changes of single molecules. Annu. Rev. Biophys. Biomol. Struct. 36, 349-369. doi: 10.1146/annurev.biophys.36.040306.132700

Tsien, R. Y. (1998). The green fluorescent protein. Annu. Rev. Biochem. 67, 509-544. doi: 10.1146/annurev.biochem.67.1.509

van Sark, W. G. J. H.M., Frederix, P. L. T. M., Bol, A. A., Gerritsen, H. C., and Meijerink, A. (2002). Blueing, Bleaching, and Blinking of Single
CdSe/ZnS Quantum Dots. ChemPhysChem 3, 871-879. doi: 10.1002/14397641(20021018)3:10<871::AID-CPHC871>3.0.CO;2-T

van Sark, W. G. J. H.M., Frederix, P. L. T. M., den Heuvel, D. J. V., Gerritsen, H. C. J., Bol, A. A., van Lingen, J. N. J., et al. (2001). Letter photooxidation and photobleaching of single CdSe/ZnS Quantum dots probed by roomtemperature time-resolved spectroscopy. Phys. Chem. B 105, 8281-8284. doi: $10.1021 / j p 012018 \mathrm{~h}$

Wang, E. C., and Wang, A. Z. (2014). Nanoparticles and their applications in cell and molecular biology. Integr. Biol. 6, 9-26. doi: 10.1039/c3ib40165k

Watanabe, T. M., Fujii, F., Jin, T., Umemoto, E., Miyasaka, M., Fujita, H., et al. (2013). Four-dimensional spatial nanometry of single particles in living cells using polarized quantum rods. Biophys. J. 105, 555-564. doi: 10.1016/j.bpj.2013.07.001

Watanabe, T. M., Fukui, S., Jin, T., Fujii, F., and Yanagida, T. (2010). Real-time nanoscopy by using blinking enhanced quantum dots. Biophys. J. 99, L50-L52. doi: 10.1016/j.bpj.2010.07.036

Watanabe, T. M., and Higuchi, H. (2007). Stepwise movements in vesicle transport of HER2 by motor proteins in living cells. Biophys. J. 92, 4109-4120. doi: 10.1529/biophysj.106.094649

Watanabe, T. M., Sato, T., Gonda, K., and Higuchi, H. (2007). Three-dimensional nanometry of vesicle transport in living cells using dual-focus imaging optics. Biochem. Biophys. Res. Commun. 359, 1-7. doi: 10.1016/j.bbrc.2007.04.168

Wells, N. P., Lessard, G. A., Goodwin, P. M., Phipps, M. E., Cutler, P. J., Lidke, D. S., et al. (2010). Time-resolved three-dimensional molecular tracking in live cells. Nano Lett. 10, 4732-4737. doi: 10.1021/nl103247v

Wells, N. P., Lessard, G. A., and Werner, J. H. (2008). Confocal, three-dimensional tracking of individual quantum dots in high-background environments. Anal. Chem. 80, 9830-9834. doi: 10.1021/ac8021899

Werver, G. (1953). Rotational Brownian motion and polarization of the fluorescence of solutions. Adv. Protein Chem. 8, 415-459. doi: 10.1016/s00653233(08)60096-0

Wombacher, R., and Cornish, V. W. (2011). Chemical tags: applications in live cell fluorescence imaging. J. Biophotonics. 4, 391-402. doi: 10.1002/jbio.201100018

Wysocki, L. M., and Lavis, L. D. (2011). Advances in the chemistry of small molecule fluorescent probes. Curr. Opin. Chem. Biol. 15, 752-759. doi: 10.1016/j.cbpa.2011.10.013

Yajima, J., Mizutani, K., and Nishizaka, T. (2008). A torque component present in mitotic kinesin Eg5 revealed by three-dimensional tracking. Nat. Struct. Mol. Biol. 15, 1119-1121. doi: 10.1038/nsmb.1491

Yildiz, A., Forkey, J. N., McKinney, S. A., Ha, T., Goldman, Y. E., and Selvin, P. R. (2003). Myosin V walks hand-over-hand: single fluorophore imaging with 1.5-nm localization. Science 300, 2061-2065. doi: 10.1126/science. 1084398

Yildiz, A., and Selvin, P. R. (2005). Fluorescence imaging with one nanometer accuracy: application to molecular motors. Acc. Chem. Res. 38, 574-582. doi: $10.1021 / \mathrm{ar} 040136 \mathrm{~s}$

Zhang, A., Dong, C., Liu, H., and Ren, J. (2013). Blinking behavior of CdSe/CdS quantum dots controlled by alkylthiols as surface trap modifiers. J. Phys. Chem. C, 117, 24592-24600. doi: 10.1021/jp408544x

Zhang, Y., and Clapp, A. (2011). Overview of stabilizing ligands for biocompatible quantum dot nanocrystals. Sensors 11, 11036-11055. doi: 10.3390/s111211036

Conflict of Interest Statement: The authors declare that the research was conducted in the absence of any commercial or financial relationships that could be construed as a potential conflict of interest.

Received: 05 June 2014; paper pending published: 18 June 2014; accepted: 05 July 2014; published online: 29 July 2014.

Citation: Ichimura T, Jin T, Fujita H, Higuchi H and Watanabe TM (2014) Nano-scale measurement of biomolecules by optical microscopy and semiconductor nanoparticles. Front. Physiol. 5:273. doi: 10.3389/fphys.2014.00273

This article was submitted to Systems Biology, a section of the journal Frontiers in Physiology.

Copyright @ 2014 Ichimura, Jin, Fujita, Higuchi and Watanabe. This is an openaccess article distributed under the terms of the Creative Commons Attribution License (CC BY). The use, distribution or reproduction in other forums is permitted, provided the original author(s) or licensor are credited and that the original publication in this journal is cited, in accordance with accepted academic practice. No use, distribution or reproduction is permitted which does not comply with these terms. 